\title{
La calidad del resumen en las publicaciones científicas españolas: estudio de caso
}

\author{
Luis Miguel Moreno Fernández \\ María Elena del Baño Aledo \\ Universidad de Murcia (España)
}

\subsection{Resumen}

Estudio de caso sobre el grado de cumplimiento de las recomendaciones y normas prescritas por las revistas científicas sobre resúmenes de autor. Para elaborar el modelo y proceder a su evaluación, se ha escogido como muestra de estudio dos publicaciones científicas españolas pertenecientes, respectivamente, a las áreas de las Ciencias de la Salud y Biblioteconomía y Documentación durante el año 2000, con el objeto de facilitar la comparación entre autores y editores con diferente grado de familiaridad con las técnicas del resumen documental. Las revistas son Fisioterapia y la Revista Española de Documentación Científica. El modelo parte de las recomendaciones propuestas por la norma UNE 50-103-90 y las específicas de las revistas; y estudia el tipo, presentación y estilo de los resúmenes, y la presencia o no de palabras clave o descriptores libres. Los resultados muestran la presencia de abundantes datos secundarios y carencias de claridad y precisión. Además, la extensión y estructura de los resúmenes no se corresponden con el contenido sustancial de los artículos. Se recomienda proseguir la investigación del tipo de estudios que publican las revistas científicas españolas y analizar hasta qué punto se encuentran preparadas para actuar de lanzadera de la investigación española hacia la comunidad científica internacional.

Palabras clave: Resúmenes. Revistas científicas. Norma UNE 50-103-90. Calidad. Normas. Evaluación.

\section{Abstract}

Case study on the degree of fulfillment of the recommendations and norms prescribed by scientific magazines on author abstracts. The evaluation model is based on the standard UNE 50-103-90, and the sample consists of the articles published during the year 2000 in two Spanish scientific publications about, respectively, Health Sciences and Library and Information Science: Fisioterapia and the Revista Española de Documentación Científica. The type, presentation

Scire. $10: 1$ (en.-jun. 2004) 91-100. 
and style of the abstracts, and the presence or not of free key words are studied. The results show the presence of abundant secondary data, and deficiencies of clarity and precision. In addition, the extension and structures of the summaries do not correspond with the substantial content of the articles. It is recommended that a investigation on the type of the studies published in Spanish scientific magazines and their suitability is carried.

Keywords: Abstracts. Scientific journals. UNE 50-103-90. Quality. Standards. Evaluation

\section{Objetivos}

La preocupación fundamental de las investigaciones y estudios sobre resúmenes científicos no es otra que la de asegurar la calidad de su contenido informativo. Desde la perspectiva del tratamiento documental de contenido (Izquierdo, 1993), que comprende la indización y el resumen de los documentos, la finalidad de estas operaciones es conseguir que el resumen sea en verdad un documento realmente representativo del documento primario, de modo tal que permita una primera aproximación comprensiva a la literatura científica rápida y fiable. Las publicaciones científicas (Delgado, 1997) han de ser las primeras interesadas en los resultados de estos estudios, pues su calidad, aparte de otros indicadores, también viene dada por la conformidad de los resúmenes que encabezan los artículos a las normas que rigen para su elaboración, dando por supuesto que las pautas dimanadas de aquéllas responden a los intereses de los usuarios y reflejan lo que ellos esperan encontrar en los resúmenes.

Entre los diversos criterios (Hartley, 1996, 1998, 2000; Pinto, 1999) susceptibles de adoptarse para enjuiciar la calidad del resumen como producto documental, nosotros hemos optado por valorar el grado de cumplimiento que hacen sus autores de la Norma UNE 50-103-90: Preparación de resúmenes y de las directrices propuestas para la redacción de resúmenes en las propias publicaciones.

\section{Método}

Para este estudio de caso hemos revisado los resúmenes que aparecen en dos publicaciones científicas españolas durante el año 2000, una del área de las Ciencias de la Salud y otra de las Ciencias de la Documentación: Fisioterapia y la Revista Española de Documentación Científica. Escogimos ambas publicaciones porque son de ámbito nacional y proporcionan a los investigadores normas extensas. En el caso de REDOC, además, hay que tener en cuenta que es la revista de mayor difusión entre los investigadores españoles en Documentación; y que acoge y publica las normas que sobre Documentación edita la Asociación Española de Normalización y Certificación (AENOR), que son traducciones y adaptaciones a nuestra lengua de las normas de la International Organization

Scire. $10: 1$ (en.-jun. 2004) 91-100. 
for Standardization (ISO). Esto permite comparar las similitudes y diferencias existentes entre los textos elaborados por una revista especializada en el ámbito documental con los que aparecen en otra publicación profesional, pero que no está especializada en Documentación, y, por lo tanto, a priori, menos familiarizada en el manejo y preparación de productos documentales.

Partiendo de esas directrices, cada resumen se analiza aplicando el modelo (Moreno Fernández y Baño Aledo, en prensa) propuesto en la tabla I, cuya finalidad es reflejar el nivel de adecuación de los resúmenes analizados a las pautas marcadas por las publicaciones periódicas. Asimismo, constituye el punto de partida para contrastar entre sí la estructura de los textos de las dos revistas analizadas, poniendo de relieve sus similitudes y diferencias respecto de las normas mencionadas.

\begin{tabular}{|c|c|c|c|c|c|c|c|c|c|c|c|c|}
\hline \multirow{3}{*}{$\begin{array}{l}\mathrm{N}^{\circ} \text { de } \\
\text { resumen } \\
\text { y revista } \\
\text { a la que } \\
\text { pertenece }\end{array}$} & \multicolumn{12}{|c|}{ Recomendaciones para la preparación de resúmenes - Norma UNE 50-103-90 } \\
\hline & \multicolumn{4}{|c|}{ Contenido informativo } & \multicolumn{6}{|c|}{ Presentación y estilo } & \multicolumn{2}{|c|}{$\begin{array}{c}\text { Palabras } \\
\text { clave o } \\
\text { descriptores }\end{array}$} \\
\hline & 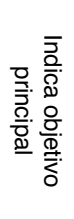 & 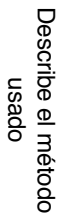 & 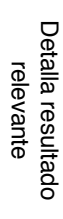 & 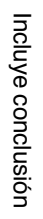 & 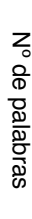 & 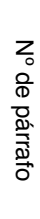 & 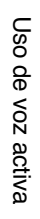 & 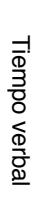 & 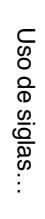 & 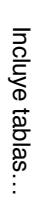 & $z_{0}$ & 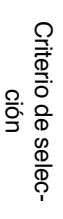 \\
\hline & & & & & & & & & & & & \\
\hline & & & & & & & & & & & & \\
\hline
\end{tabular}

Tabla I. Tipo, estructura y presentación de los resúmenes analizados

Aplicando el modelo propuesto obtenemos cinco tipos resúmenes: a) informativos puros, que incluyen objetivos, método, resultados y conclusiones; b) informativos orientados hacia los resultados, que contienen objetivos, método y resultados; c) informativos orientados hacia las conclusiones, que describen objetivos, método y conclusiones; d) indicativo puro, que incluye objetivos y método; y e) indicativo sencillo, que admite sólo los objetivos. Los resúmenes mixtos describen los objetivos y las conclusiones. Mientras que un resumen se considera indicativo sencillo cuando incluye sólo el primer apartado de los que se consideran relevantes en un trabajo científico, es decir, el objetivo u objetivos del trabajo de investigación. Sin embargo, en el presente estudio se han incluido dentro de los resúmenes indicativos sencillos aquéllos que, además, ofrecen otro tipo de información considerada en principio menos relevante, como antecedentes históricos, hipótesis, redundancias, introducciones al tema... De hecho, la mayoría de ellos 
rara vez contiene exclusivamente el objetivo del estudio e incluso en alguno es difícil localizarlo entre toda la información superficial que posee.

Para la presentación y estilo hemos procedido a tabular los requisitos propuestos por la Norma UNE 50-103-90: extensión; número de párrafos en los que se divide el texto; uso de voz activa; tiempo verbal; tratamiento de acrónimos, siglas o abreviaturas y de tablas, ecuaciones, diagramas o formas estructurales. También se ha evaluado la existencia de palabras clave y su incardinación o no en el resumen y título del trabajo presentado.

\section{Resultados}

Los resúmenes analizados son 43 . De ellos, el $63 \%$ pertenecen a la revista de Fisioterapia y el $37 \%$ a REDOC. Considerados en conjunto los textos de ambas revistas, observamos que la proporción de resúmenes indicativos $(60 \%)$ supera con creces a la de resúmenes informativos (33\%), llegando a duplicarla. En el otro extremo están los resúmenes mixtos, presentes en escasa proporción $(7 \%)$.

El análisis de cada una de las revistas por separado (figura 1) revela una proporción similar entre resúmenes indicativos e informativos, pero se aprecia que los resúmenes mixtos sólo figuran en la revista Fisioterapia. Sin embargo, en esta revista el resumen informativo cuenta, sorprendentemente, con escasa representación: a pesar de las expresas prescripciones de la publicación, no llegan al $30 \%$ del total. Una clasificación más exhaustiva de los 43 resúmenes (figura 2) muestra la predilección de los autores, en general, por los resúmenes indicativos

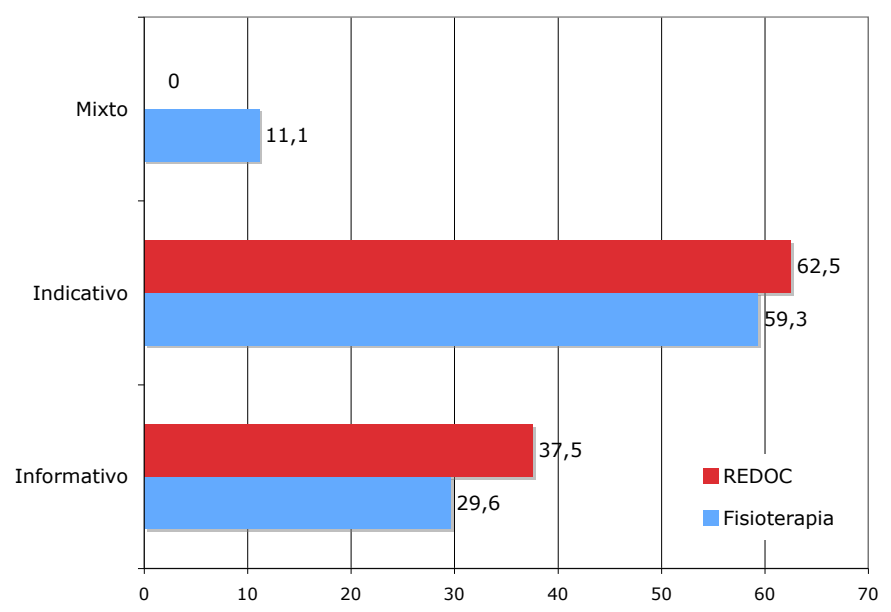

Figura 1. Distribución de los tipos de resumen en las revistas Fisioterapia y REDOC en el año 2000

Scire. $10: 1$ (en.-jun. 2004) 91-100. 


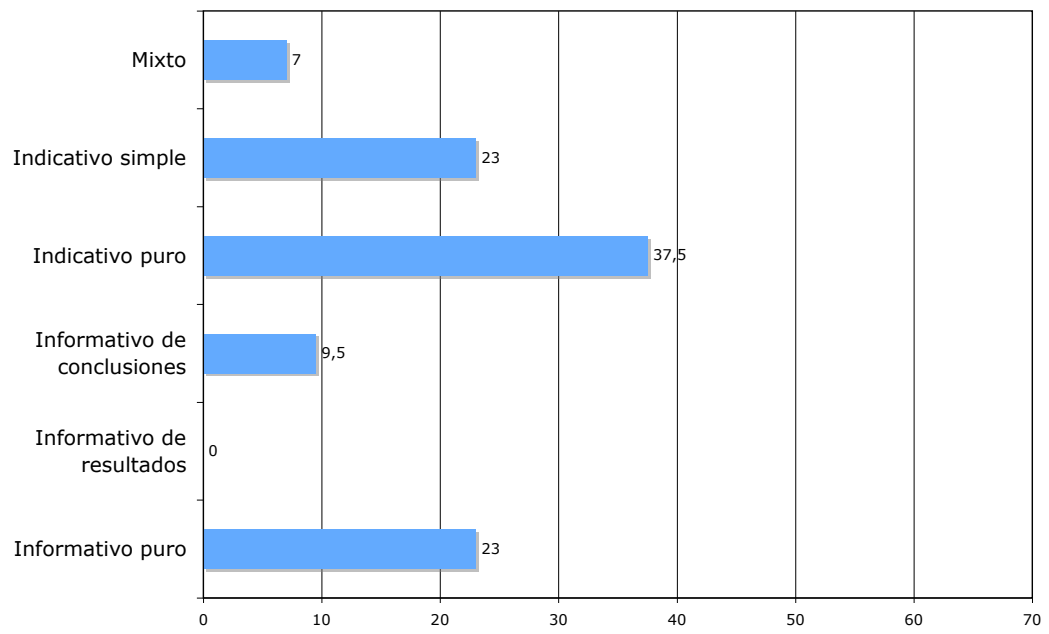

Figura 2. Tipos de resúmenes representados en Fisioterapia y REDOC

que señalan el objetivo del trabajo y explican el método, seguidos de los indicativos sencillos e informativos puros. Los resúmenes informativos orientados hacia las conclusiones se usan más que aquéllos orientados hacia los resultados, que no aparecen representados en esta muestra.

La figura 3 contiene esta misma clasificación para cada una de las revistas. Las dos mantienen aproximadamente la misma proporción de la muestra total,

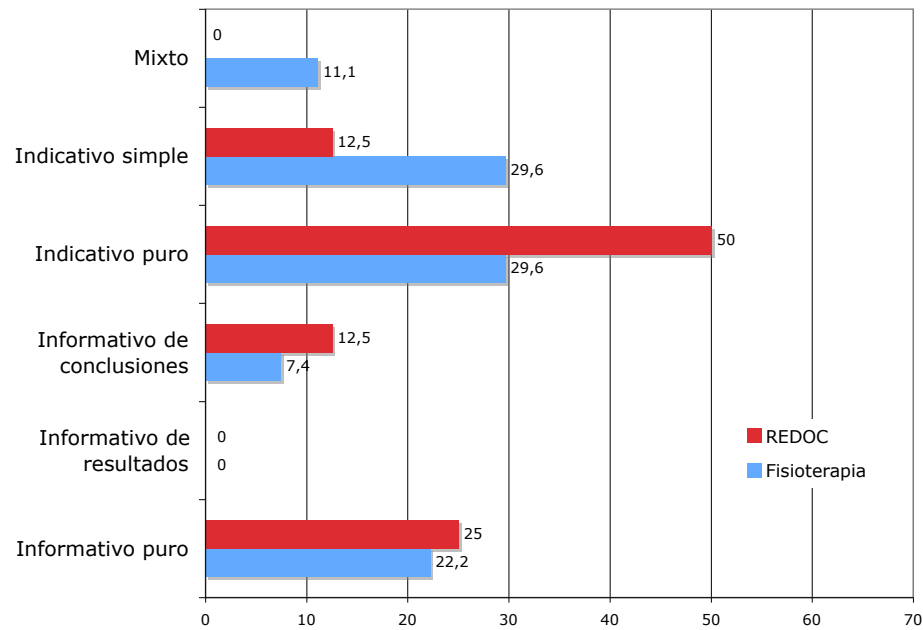

Figura 3. Comparativa de los tipos de resúmenes por revista

Scire. $10: 1$ (en.-jun. 2004) 91-100. 


\section{6}

es decir, sigue predominando el resumen indicativo sobre el informativo. Los resúmenes mixtos son muy escasos en Fisioterapia e inexistentes en REDOC. La revista Fisioterapia contiene el mismo número de resúmenes puros y sencillos, mientras que en $R E D O C$ los primeros superan ampliamente al resto. En la revista Fisioterapia se solicita con más detalle que en REDOC el contenido de los resúmenes, y, sin embargo, la proporción de resúmenes indicativos es mucho mayor, lo que quizá pueda atribuirse fundamentalmente a un factor: que los resúmenes indicativos están reflejando la escasa representación de trabajos empíricos en beneficio de estudios descriptivos, cuya estructura no se adapta a la del resumen de carácter informativo. REDOC también abusa de los resúmenes indicativos, pero no de los indicativos sencillos. Estos últimos pueden considerarse los resúmenes más deficientes por su escaso valor informativo y por haber incluido entre ellos a todos los resúmenes con escasa información substancial, en beneficio de una abundante información superficial y accesoria. Esto obedece a que los autores de los resúmenes que aparecen en esta revista tienen más conocimiento de la técnica del resumen que los de Fisioterapia.

En cuanto a la presentación, para la revista Fisioterapia la media de palabras por resumen es de 140, con un mínimo de 45 y un máximo de 362 , mientras que en la revista $R E D O C$, la media está situada en 141 palabras por resumen, con una cota inferior de 42 y una cota superior de 232. Los resúmenes de REDOC muestran una dispersión menor del número de palabras por resumen que la revista Fisioterapia. Por lo que a la longitud se refiere, esto indica una mayor homogeneidad en la presentación de los resúmenes en la primera que en la segunda. La cota superior de palabras por resumen en la revista Fisioterapia supera en mucho las recomendaciones de la norma UNE, y, en el caso de REDOC, aunque su cota superior no excede la recomendación de la mencionada norma, sí supera las prescripciones de la propia publicación. La dispersión del número de palabras por resumen de la revista Fisioterapia afecta a los tres grandes tipos de resumen, mientras que en REDOC se mantienen unos rangos más adecuados a las características estructurales de la mayoría de los resúmenes representados.

El exceso de resúmenes indicativos sencillos en Fisioterapia es el motivo de que existan muchos de ellos con un número de palabras pequeño (menor de 100). En las recomendaciones de Fisioterapia no se especifica el número de palabras por resumen; y esto también es una de las causas de que haya tanta heterogeneidad y coincidan, en la misma revista, resúmenes de 45 palabras con otros de 362, por ejemplo. La revista Fisioterapia presenta el artículo y el resumen de los trabajos en un texto a dos columnas. Habitualmente, el resumen ocupa la primera página pero, cuando el número de palabras por resumen excede aproximadamente de las 220, es preciso pasar a la página siguiente. Esto dificulta la lectura rápida

Scire. $10: 1$ (en.-jun. 2004) 91-100. 
del texto, y, por lo tanto, también ralentiza la identificación de las zonas de interés que pueda haber para el lector.

Por lo que se refiere a la organización o estructuración de la información en el texto, se observa que en la revista Fisioterapia se incumple la norma de estructurar el resumen en un único párrafo en algo más del $50 \%$ de los casos, en tanto que el porcentaje de incumplimiento en $R E D O C$ es del $12,5 \%$. Es posible que esto se deba a que los trabajos contenidos en esta revista son experimentales y su presentación es más clarificadora si cada uno de sus elementos integrantes recogidos en el resumen se separa en párrafos independientes. En cualquier caso, es inadecuado para la calidad de los resúmenes que este tipo de "literaturas" incumpla las directrices concernientes a su presentación en un solo párrafo, porque eso no facilita su legibilidad. Dada la longitud media de los resúmenes, que debería ser más homogénea, sería mejor que se exigiera con rigor la presentación del texto en un párrafo íntegro. Sin embargo, la presentación estructurada es aceptable y aun recomendable en los resúmenes informativos, siempre que cada uno de los elementos constituyentes conforme un párrafo en sí mismo, dado que el lector percibe de un vistazo dónde figura el elemento o elementos que le interesan. Lo que resulta inadecuado es que un determinado elemento aparezca escrito en más de un párrafo, en la medida en que desorienta al lector.

En cuanto al estilo, tres de los resúmenes de REDOC usan siglas o acrónimos sin expresar primero su significado. Esto ocurre en dos de los resúmenes de Fisioterapia. La cifra no es muy significativa, pero no tendría que haber ningún caso. Los autores de estos resúmenes no usan la voz pasiva. Y en lo tocante al tiempo verbal empleado en la revista $R E D O C$ se emplea el tiempo pretérito en seis de los 16 resúmenes, eso sí, siempre usando la forma impersonal. En la revista Fisioterapia el uso del pretérito ocurre en dos de los 27 resúmenes de la revista. En conjunto, beneficia la calidad de los resúmenes que éstos se hallen redactados en voz activa y en tiempo presente. Una y otro propician que la lectura de esta clase de géneros literarios sea más ágil y directa. Por otro lado, que no se empleen simultáneamente en este contexto el presente y el pretérito evita confusiones, por más que la utilización del tiempo pasado esté autorizada para destacar los aspectos metodológicos de la labor efectuada, no así para las conclusiones y los objetivos. Los resúmenes no incluyen tablas o diagramas en el texto, ni hay tampoco referencias a los que pudiera haber en el cuerpo o apéndices de los documentos resumidos, a pesar de no ser esta una recomendación expresa en ninguna de las dos publicaciones.

La media de palabras clave en los resúmenes de Fisioterapia es de cuatro, aunque la moda es inferior (tres en los ocho resúmenes). En REDOC los resúmenes ofrecen una media de cinco palabras clave por resumen y la moda asciende a seis. En los resúmenes de Fisioterapia hay más variedad en la proporción de

Scire. $10: 1$ (en.-jun. 2004) 91-100. 
palabras clave con las que se identifica el documento, mientras que este elemento es más heterogéneo en REDOC. Es decir, que hay menos oscilaciones en la proporción de descriptores libres en esta última revista. La proporción de vocablos o expresiones escogidas para la indización que aparecen incardinadas en el cuerpo del resumen y en el título representa el 65,23\% de los términos en la revista Fisioterapia y el $53 \%$ en REDOC. Son porcentajes elevados, quizá porque el número medio de descriptores utilizados en la indización es reducido. De todos modos, es positivo que la proporción de descriptores libres empleados para indizar que aparecen en los textos sea elevada, porque ésto redunda en beneficio de las búsquedas en lenguaje natural, incrementando la tasa de llamada. Es por eso por lo que la inclusión de las palabras clave en el cuerpo del resumen puede considerarse un indicador de calidad. Pero los descriptores libres que aparecen sólo en el texto del resumen no arrojan porcentajes muy elevados: en la revista Fisioterapia el porcentaje es del 22,22\%, frente al 12,5\% de REDOC.

\section{Conclusiones}

Muchos de los resúmenes de este estudio contienen datos secundarios; son resúmenes carentes de calidad y precisión, factores que impiden al lector decidir si merece la pena recuperar o no el artículo completo en función de sus intereses. Las indicaciones de ambas revistas para la presentación de resúmenes señalan las partes del trabajo a las que han de hacer referencia, pero esto parece insuficiente. También convendría aclarar qué elementos no deben entrar en el resumen. Por ejemplo, los autores tienen que saber que no han de introducir en los resúmenes antecedentes históricos, ni argumentaciones encaminadas a justificar la realización de su trabajo, etc. Y dejar bien claros estos extremos es obligación de la propia revista, cuya política de publicación habría de asumir la formulación de unas directrices claras - no ambiguas - acerca de los distintos tipos de resúmenes que encabezan los diferentes trabajos que se acogen en sus páginas.

Los resúmenes tienen que adaptarse al tipo de trabajo realizado: es decir, los trabajos de investigación precisan de resúmenes científicos que contengan todos los apartados principales de un trabajo experimental; pero deben adaptar su estructura $-y$, por ende, la de los elementos que ofrecen - a otros trabajos de naturaleza más descriptiva, que no contienen algunos de los constituyentes habituales de la investigación empírica. La revista Fisioterapia considera esta distinción, no así REDOC. En ningún caso es admisible la introducción en los resúme-

nes de conclusiones "ficticias", que no guardan relación con el tema estudiado, o que plantean hipotéticos logros futuros que acaso se deriven del tema analizado. Este inconveniente se obviaría si las publicaciones ofreciesen la posibilidad de presentar diferentes modelos de resúmenes para cada uno de los géneros cien-

Scire. $10: 1$ (en.-jun. 2004) 91-100. 
tíficos acogidos en sus páginas: contribuciones originales, revisiones, informes breves, comunicaciones importantes, comentarios, ensayos, etc.

Aunque hay que ampliar la muestra objeto de estudio para llegar a resultados más concluyentes, puede plantearse la hipótesis de que tanto la extensión de los resúmenes como la división en párrafos no responden a los requerimientos del contenido sustancial de los trabajos. La articulación de los resúmenes en párrafos resulta positiva siempre y cuando cada uno de los elementos integrantes constituya un solo párrafo, porque esto permite al usuario seleccionar la información que le interesa y decidir si precisa o no leer el texto íntegro. De lo contrario, es decir, cuando se entremezclan en el párrafo elementos distintos, el usuario se confunde y pierde tiempo en desbrozar dónde se halla la información que le interesa.

El número de términos o palabras clave que acompañan a los resúmenes se ajusta a las recomendaciones de la norma UNE. Pero habría que preguntarse en qué grado representan los aspectos más significativos del documento. Tal vez debiera sugerirse a los autores que indizaran sus documentos recurriendo a determinadas fuentes o lenguajes documentales especializados en sus respectivas áreas de conocimiento.

En nuestra opinión, el resumen que encabeza los trabajos que ven la luz en las revistas científicas constituye un indicador más de la calidad de éstas y de las contribuciones científicas que acogen. Es por eso por lo que merece la pena procurar que sean mejores de lo que en la actualidad son en muchas de ellas. A la vista de estos primeros resultados, parece interesante investigar qué tipo de estudios se publican con más frecuencia en las publicaciones periódicas españolas y en qué modo éstas se encuentran preparadas para actuar de lanzadera de la investigación científica española a la comunidad científica internacional.

\section{Referencias}

Delgado López-Cózar, Emilio (1997). Evaluación y aplicación de las normas de presentación de publicaciones periódicas: revisión bibliográfica. // Revista Española de Documentación Científica (REDOC). 20:1 (1997) 39-51.

Izquierdo Arroyo, José María (1993). De la semiótica del discurso a la semiótica documental. // Moreiro González, José Antonio. Aplicación de las ciencias del texto al resumen documental. Madrid: Boletín Oficial del Estado; Universidad Carlos III, 1993.

Hartley, James; Sydes, Matthew (1996). Which layout do you prefer?: An Analysis of readers preferences for different typografic layouts of structured abstracts. // Journal of Information Science. 22: 1 (1996) 27-37.

Hartley, James (1998). Is it appropiate to use structured abstracts in non-medical sciences journals?. // Journal of Information Science. 24: 5 (1998) 359-364.

Scire. $10: 1$ (en.-jun. 2004) 91-100. 
Hartley, James (2000). Are structured abstracts more or less accurate than traditional ones?: a study in the psicological literature. // Journal of Information Science. 26: 4 (2000) 273-277.

Moreno Fernández, Luis Miguel; Baño Aledo, María Elena del (en prensa). La aplicación de la Norma UNE 50-103-90 para la preparación de resúmenes en las revistas "Fisioterapia" y "Revista Española de Documentación Científica (REDOC)": modelo para evaluar la calidad de los resúmenes de autor que aparecen en las publicaciones periódicas. (En prensa).

Pinto, María; Lancaster, F.W. (1999). Abstracts and Abstracting in Knowledge Discovery. // Library Trends. 48: 1 (1999) 234-248. 